\title{
Application of percutaneous foraminotomy with a specially designed drill tip for foraminal stenosis patient \\ - A case report -
}

Received November 10, 2017

Revised 1st, January 4, 2018 2nd, May 1, 2018

Accepted May 1, 2018

\section{Corresponding author}

Sung-Eun Sim, M.D., Ph.D. Department of Anesthesiology and Pain Medicine, SMG-SNU Boramae Medical Center, 20 Boramae-ro 5-gil, Dongjak-gu, Seoul 07061, Korea Tel: 82-2-870-2114

Fax: 82-2-831-2826

E-mail: euny62827@hanmail.net ORCID

http://orcid.org/0000-0002-6643-0172

\section{Hyunjung Choo and Sung-Eun Sim}

Department of Anesthesiology and Pain Medicine, SMG-SNU Boramae Medical Center, Seoul, Korea

This case report describes a new method of pain management intervention: percutaneous foraminotomy using the Claudicare system (Seawon Meditech, Korea). In this case, a 77-year-old Asian man visited the hospital with motor weakness in his left foot. He was diagnosed with L4-5 grade three foraminal stenosis using Magnetic Resonance Imaging on both sides. A left L4-5 foraminal decompression was performed using percutaneous foraminotomy. The patient revisited the hospital after 17 months because the same symptoms recurred in his right foot. We observed that the symptoms on the left foot had disappeared completely. We confirmed the lesion on the right side and the postoperative change on the left side on the magnetic resonance imaging (MRI) image. Both the pre- and postoperative MRI images were compared by measuring the dimensions of the foraminal area (28.12 $\mathrm{mm}^{2}$ vs. $38.58 \mathrm{~mm}^{2}$, repectively). T1W images showed signs of increased epidural soft tissue after percutaneous foraminotomy.

Key Words: Foraminotomy, Spinal stenosis, Spine.
Degenerative diseases are rapidly increasing all over the world with the increase of the elderly population.

Spinal stenosis is one of the common degenerative disease and various treatment methods have been applied to treat this disease and control the pain. Currently, diverse, non-operative interventions used for the treatment of spinal stenosis in addition to novel interventional techniques and tools are rapidly [1].

These interventions are alternative methods to patients who are not responsive to conservative treatment like physical therapy, medications and epidural steroid injections. Furthermore, these can be useful treatment options to patients who refuse surgery.

Leg pain while walking, which is relieved when the patient squats, sits, or rests, is a typical symptom of foraminal ste- nosis. This suggests that the pressure on the nerve root is an important factor of disease pathogenesis and that foraminal decompression is central to the treatment of foraminal stenosis [2].

The purpose of this report is to introduce a new therapeutic intervention for foraminal stenosis-percutaneous foraminotomy with the use of the Claudicare system. Percutaneous foraminotomy is a procedure that indirectly decompresses the foramen, and is performed in the safe area inside the neural foramen, which is Kambin's triangle.

\section{CASE REPORT}

A 77-year-old $164 \mathrm{~cm}, 62 \mathrm{~kg}$ previously healthy Asian man visited the hospital with grade 3 motor weakness on his left

This is an Open Access article distributed under the terms of the Creative Commons Attribution Non-Commercial License (http://creativecommons.org/licenses/by-nc/4.0) which permits unrestricted non-commercial use, distribution, and reproduction in any medium, provided the original work is properly cited. 
foot that began six months earlier. Grade three L4-5 foraminal stenosis was noted on both sides and central canal stenosis [3] was seen on magnetic resonance imaging carried out at another hospital (Fig. 1). As the patient presented with the clinical symptoms on the left, we decided to perform the percutaneous foraminotomy (Claudicare) on only the left side.

The patient was admitted on the day of operation. Prior to the procedure, an IV catheter was placed on his arm and $1.0 \mathrm{~g}$ of cefazolin was given intravenously as prophylaxis. After the informed consent was signed by the patient and received by us, he entered the operating room.

The patient was instructed to stay in a prone position on the operating table. The skin of the operative target was prepared, sterilized, and draped. The C-arm was tilted obliquely to the left, on the ipsilateral side of the area where the patient's symptoms were observed, after aligning the inferior endplates of L4 and the superior endplate of L5. Skin and subcutaneous tissue were locally anesthetized with lidocaine onto the
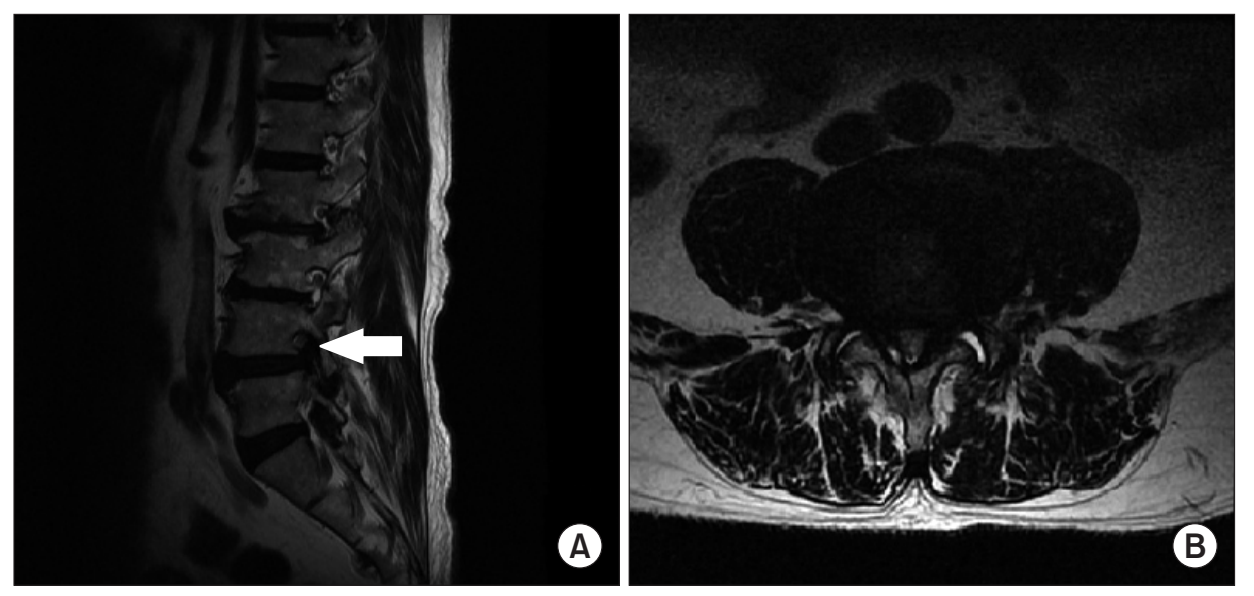

Fig. 1. Initial lumbar spine magnetic resonance imaging shows foraminal stenosis at L4-5 level. (A) Sagittal view (arrow: Left L4-5 intervertebral foramen), (B) Axial view.
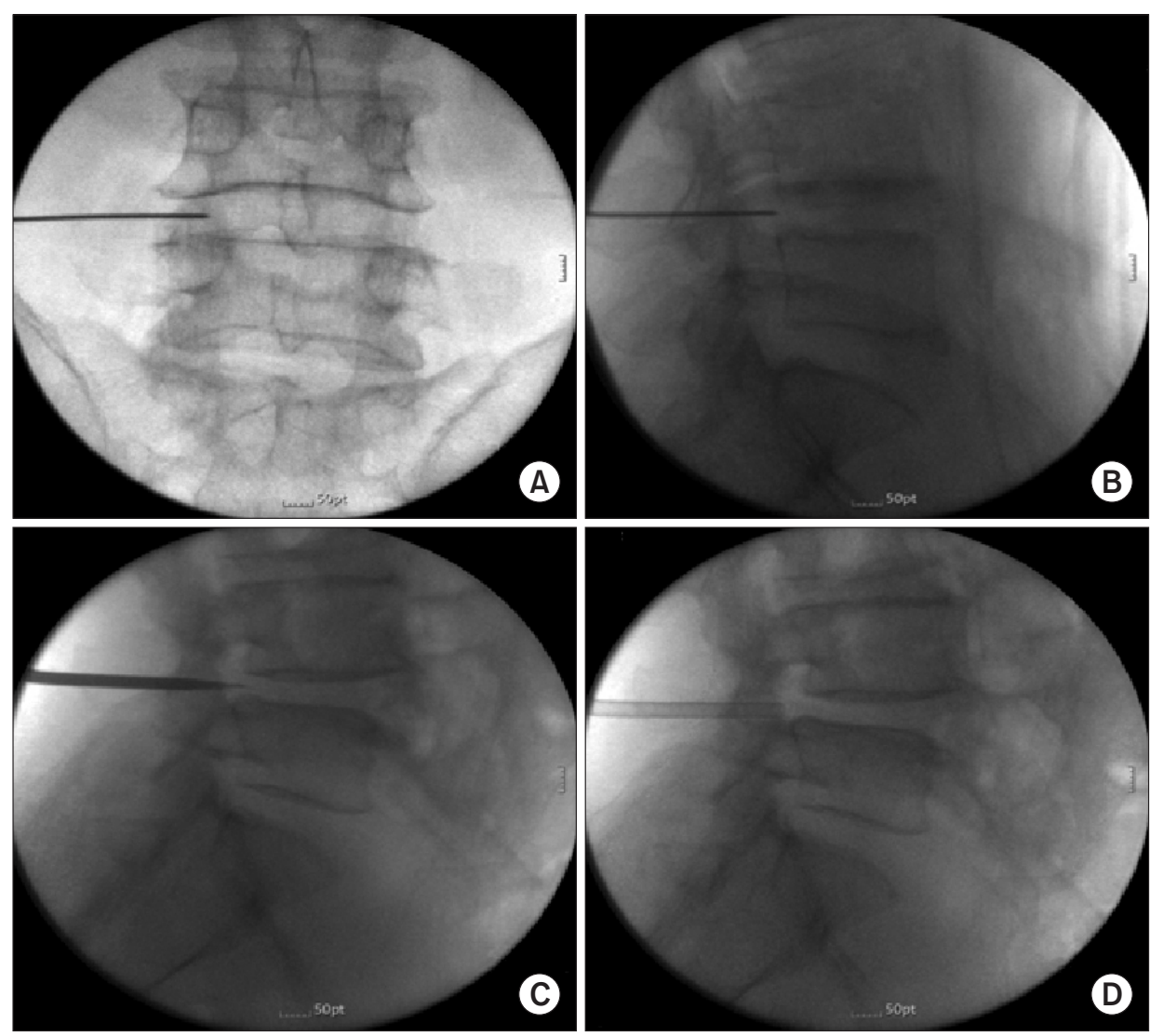

Fig. 2. Fluoroscopy images of percutaneous foraminotomy. (A) Anteroposterior view, guide wire to Kambin's triangle, (B) lateral view, guide wire to Kambin's triangle, $(C)$ lateral view, dilator insertion, and (D) lateral view, working channel insertion. 
surface of the superior articular process (SAP) of L5. An introducer needle located the Kambin's triangle (Fig. 2A and B).

After inserting the guide wire through the introducer needle, the skin canal was gently widened with a dilator. The working cannula was inserted through the dilated canal using the Seldinger maneuver and situated at Kambin's triangle (Fig. 2D).

The Claudicare (Fig. 3C) drilling tip with the bird-beakshaped shield was inserted through the working cannula and the shield was situated on the side of the affected nerve root. Decompression of the neural foramen was done through the drilling of the hypertrophied facet joint, the ligaments adjacent to the superior articular process and the bulging disc. Af-

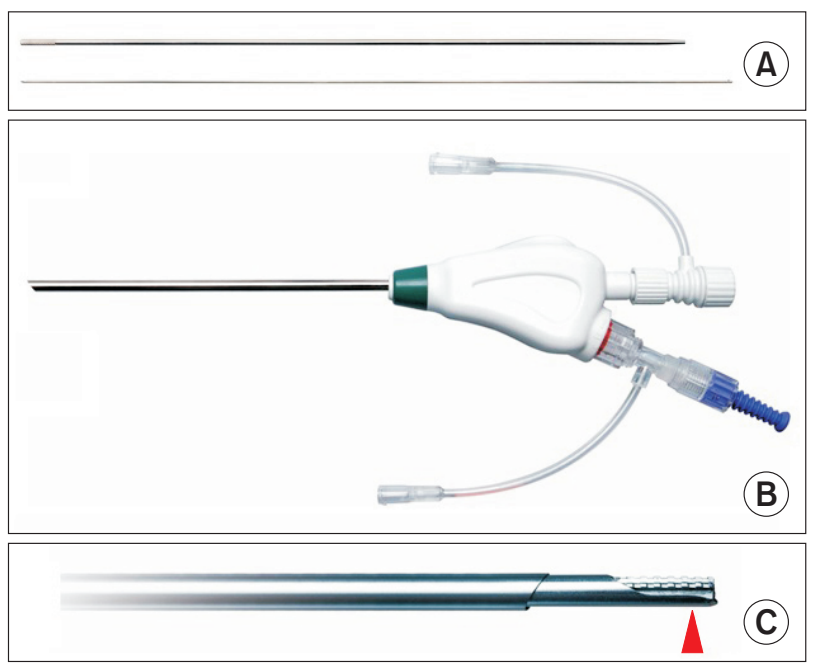

Fig. 3. Instruments for percutaneous foraminotomy: (A) Dilator, guide wire, (B) Working cannula with an epiduroscope port, (C) Claudicare with bird-beak-shaped shield (arrowhead). ter constantly checking the position of the drill on the lateral view, we proceeded to the posterior margin of the vertebral body.

The drilling tip was removed from the cannula and the drug mixture with levobupivacaine (Chirocaine), hyaluronidase, and dexamethasone was injected through the empty working cannula for pain control and adhesion prevention.

The puncture site was sutured after removing the working cannula. Afterwards, the patient was moved to the recovery room. Because he did not have any side effects, such as severe pain or motor weakness, he was discharged after two hours per routine.

The patient was scheduled for follow-up four weeks after the procedure, but was lost to follow-up.

After 17 months, he returned to the hospital with right leg pain, numbness of the right hallux, and foot drop while walking. Motor grade of left ankle recovered to grade 5 compared to before the claudicare which was grade 3 . The patient requested that we perform the same procedure using the Claudicare on the right side.

\section{MRI findings}

The neural foramen was evaluated using the Lee classification and it was diagnosed as a grade 3 foraminal stenosis in both a preoperative and postoperative magnetic resonance imaging (MRI) [3] (Figs. 1 and 4).

Both MRIs were compared. The dimensions of the foraminal area were also measured. The foraminal area was calculated after outlining the perimeter of the neural foramen

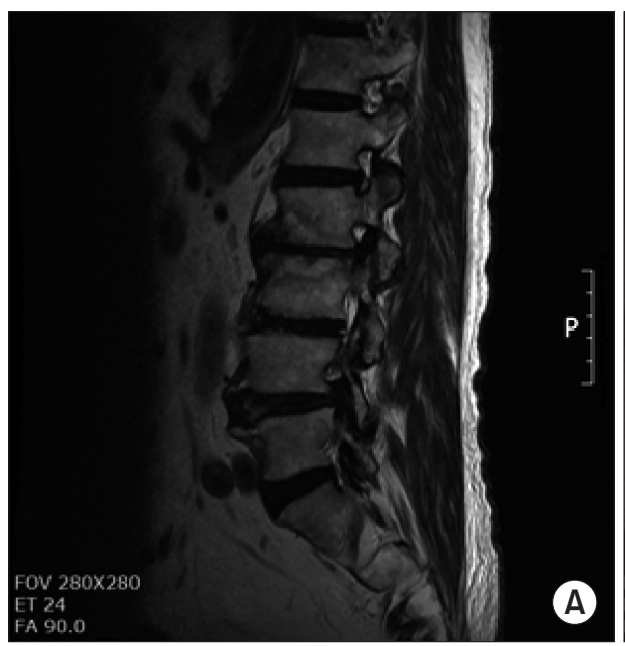

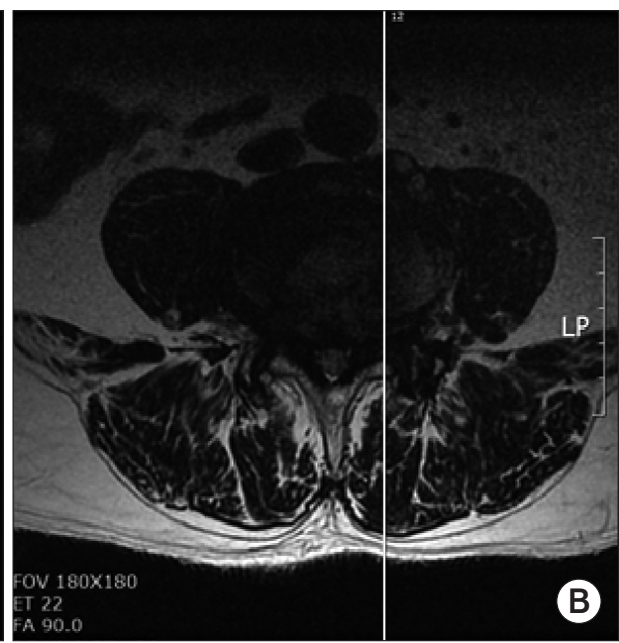

Fig. 4. Fifteen months after initial L4-5 percutaneous foraminotomy magnetic resonance imaging. (A) Sagittal view and (B) axial view. 
on parasagittal images. The adjacent superior and inferior vertebral pedicles, the posteroinferior margin of the superior vertebral body, the posterior intervertebral disc, and the posterosuperior margin of the inferior vertebral body were used as anterior boundaries with the ligamentum flavum and the superior and inferior articular facets serving as the posterior

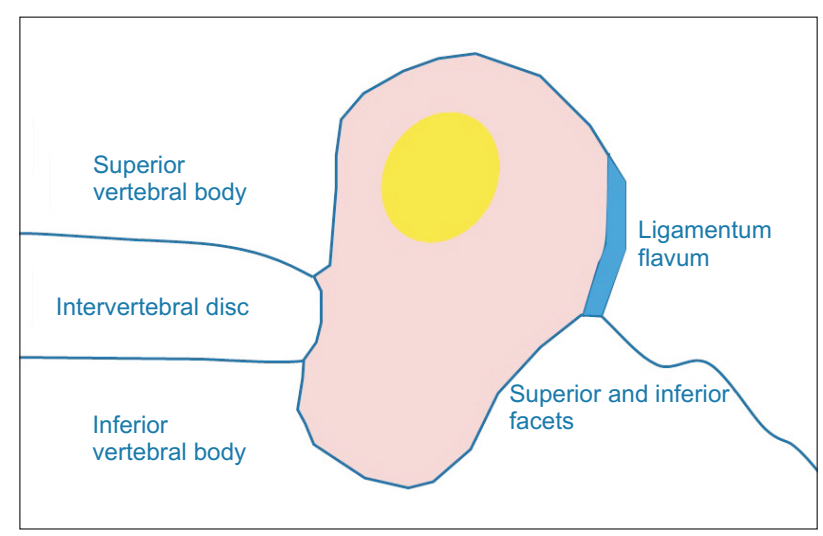

Fig. 5. Schematic image of intervertebral foramen. Red area is foraminal area that is used to calculate the dimension of foramen. boundaries [4] (Fig. 5).

The preoperative dimension of the L4-5 intervertebral foramen on the left was $28.12 \mathrm{~mm}^{2}$ whereas the postoperative dimension was $38.58 \mathrm{~mm}^{2}$, showing an increase in area (Fig. $6)$.

T1-weighted preoperative axial (Fig. 7A) and postoperative axial (Fig. 7B) images show signs of epidural soft tissue lateral to and anterolateral to the dural tube increasing after percutaneous foraminotomy. This means that there is an increased amount of soft tissue surrounding the nerve root [5].

The same procedure using Claudicare was performed for the right side foraminal stenosis lesion.

\section{DISCUSSION}

Spinal stenosis occurs when there is a narrowing of the foramen space due to a disc protrusion, a bulging disc, a hypertrophy of transforaminal ligaments (TFLs), a hypertrophy of SAP, or an osteophyte in the foramen. All these causes pressurize the nerve root which leads to the disease.
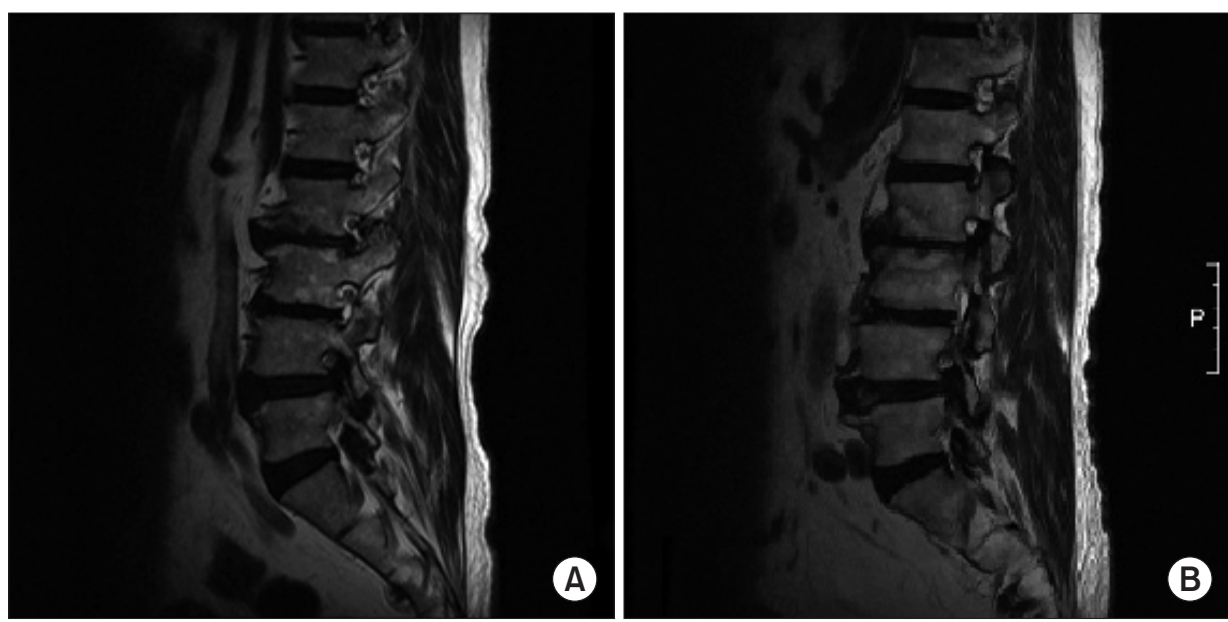

Fig. 6. T2-weighted magnetic resonance sagittal images pre- $(A)$ and post-Claudicare (B) imaging at foraminal plane.
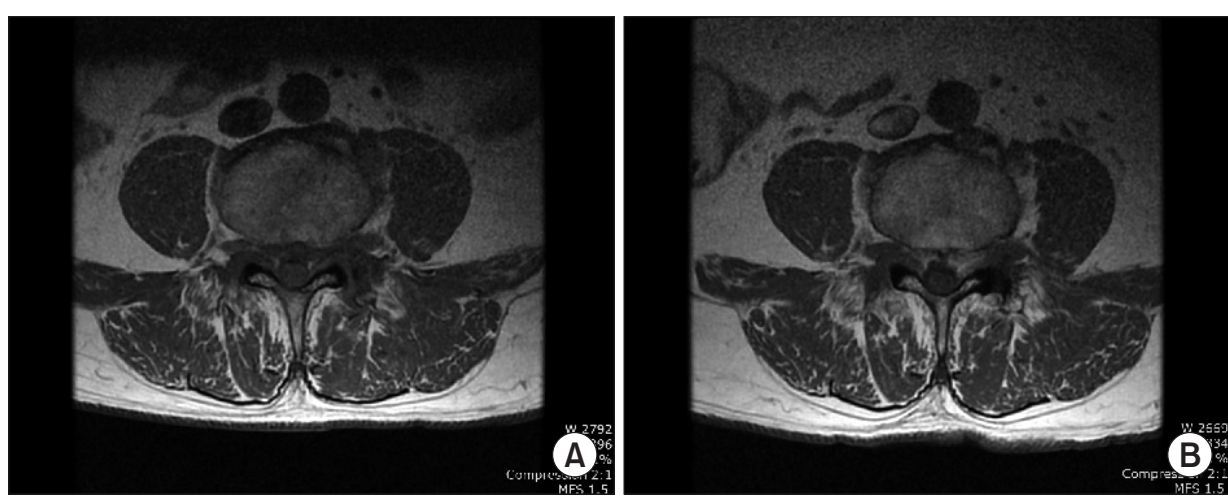

Fig. 7. T1-weighted magnetic resonance axial images pre- $(A)$ and postClaudicare (B) imaging. 
The purpose of intervention in this disease is to decrease the pressure and tension applied on the nerve by removing the source of pressure [6-12]. The percutaneous foraminotomy introduced in this case report is a newly developed minimally invasive device that reduces pressure on the nerve root as well as the blood vessels in the neural canal. The aim of this treatment is to indirectly decompress the neural canal based on the principle that claudication is temporarily relieved when patients with spinal stenosis squat.

The aims of this procedure are: 1) the elimination of thickened TFLs, 2) the grating of the ventral side of hypertrophied SAP, 3) the removal of foraminal and extraforaminal lumbar disc herniation (Fig. 8).

According to an anatomical cadaver study, a more medial access to the working zone of Kambin's triangle using foraminoplasty by virtue of grating the SAP with a surgical burr or trephine not only provides safe access to the intra-canal or foraminal space, but also makes it possible to prevent nerve injury upon exit $[13,14]$. Furthermore, this space is where the thickened TFLs to be decompressed are located. Therefore, we expect symptomatic relief after removing thickened TFLs by accessing the foramen through Kambin's triangle [14].

After widening the intervertebral foramen space, the reaction of procedures like selective transforaminal block or epidural block is considered to be better than before.

Another aspect that augments the safety of the procedure is a special drilling tip. On one side of the drilling tip, there is a shield that looks like a bird's beak. This shield plane can then

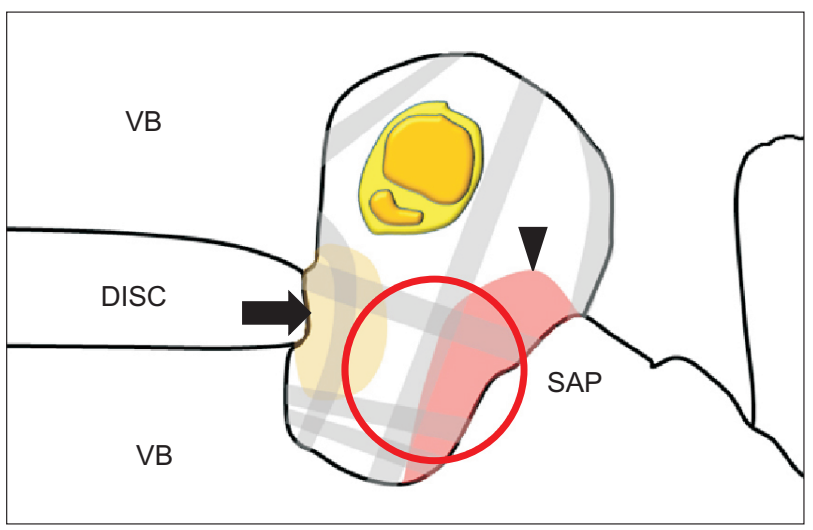

Fig. 8. Schematic image of neural foramen and target of drilling. Red circle: placement of Claudicare. Arrow: bulging disc. Arrowhead: hypertrophy of superior articular process. Gray lines: thickened transforaminal ligament filled in the foraminal space. VB: vertebral body, SAP: superior articular process. be situated on the side of the nerve root to prevent the nerve root from being damaged.

In addition, as this intervention is performed under only local infiltration, one can easily monitor the symptoms of the patient. The procedure can also be safely performed without using an endoscope unless approaching from the point where signs of irritation occur.

The simplicity of the procedure is a major advantage of percutaneous foraminotomy. Only fluoroscopy and the Claudicare system are necessary for this procedure. Thus, the number of required instruments is few, and the treatment itself is easy to perform.

There is, however, a disadvantage to the procedure in that the indications are limited. Indications of percutaneous foraminotomy include patients with short-term effects from root block, with a grade 2 or 3 lumbar foraminal stenosis, either with or without central stenosis.

It is not indicated in the case of a severe bony spur or an abnormality in spine alignment. At the L5-S1 level, approach may be extremely difficult as well, because the operative window narrows progressively because of the prominent iliac crest, wider disc space, and more oblique pedicles. In addition, the more coronally oriented facet joints at L5-S1 impede posterior or posterolateral access to the disc [15].

This case introduces a non-operative treatment method that physically expanding the intervertebral foramen to reduce pressure on the nerve root. Considering the population of elderly people is rapidly growing world-widely, number of spinal stenosis patients will increase. So, If we can treat the foraminal stenosis via nonoperative method, It will be beneficial to patients.

\section{REFERENCES}

1. Manchikanti L, Pampati V, Hirsch JA. Utilization of interventional techniques in managing chronic pain in medicare population from 2000 to 2014: an analysis of patterns of utilization. Pain Physician 2016; 19: E531-46.

2. Rydevik B, Brown MD, Lundborg G. Pathoanatomy and pathophysiology of nerve root compression. Spine (Phila Pa 1976) 1984; 9: 7-15.

3. Lee S, Lee JW, Yeom JS, Kim KJ, Kim HJ, Chung SK, et al. A practical MRI grading system for lumbar foraminal stenosis. AJR Am J Roentgenol 2010; 194: 1095-8. 
4. Singh V, Montgomery SR, Aghdasi B, Inoue H, Wang JC, Daubs MD. Factors affecting dynamic foraminal stenosis in the lumbar spine. Spine J 2013; 13: 1080-7.

5. Ross JS, Masaryk TJ, Modic MT, Bohlman H, Delamater R, Wilber G. Lumbar spine: postoperative assessment with surface-coil MR imageing. Radiology 1987; 164: 851-60.

6. Wang YP, Zhang W, Li BL, Sun YP, Ding WY, Shen Y. Suprapedicular foraminal endoscopic approach to lumbar lateral recess decompression surgery to treat degenerative lumbar spinal stenosis. Med Sci Monit 2016; 22: 4604-11.

7. Chang HS. Microsurgical posterolateral foraminotomy on patients with adult isthmic spondylolisthesis. World Neurosurg 2017; 100: 434-9.

8. Gu YT, Cui Z, Shao HW, Ye Y, Gu AQ. Percutaneous transforaminal endoscopic surgery (PTES) for symptomatic lumbar disc herniation: a surgical technique, outcome, and complications in 209 consecutive cases. J Orthop Surg Res 2017; 12: 25.

9. Lee CW, Yoon KJ, Ha SS, Kang JK. Foraminoplastic superior vertebral notch approach with reamers in percutaneous endoscopic lumbar discectomy: technical note and clinical outcome in limited indications of percutaneous endoscopic Lumbar dis- cectomy. J Korean Neurosurg Soc 2016; 59: 172-81.

10. Li ZZ, Hou SX, Shang WL, Cao Z, Zhao HL. Percutaneous lumbar foraminoplasty and percutaneous endoscopic lumbar decompression for lateral recess stenosis through transforaminal approach: technique notes and 2 years follow-up. Clin Neurol Neurosurg 2016; 143: 90-4.

11. Uniyal P, Choi G, Khedkkar B. Percutaneous transpedicular lumbar endoscopy: a case report. Int J Spine Surg 2016; 10: 31.

12. Wen B, Zhang X, Zhang L, Huang P, Zheng G. Percutaneous endoscopic transforaminal lumbar spinal canal decompression for lumbar spinal stenosis. Medicine (Baltimore) 2016; 95: e5186.

13. Xin G, Shi-Sheng H, Hai-Long Z. Morphometric analysis of the YESS and TESSYS techniques of percutaneous transforaminal endoscopic lumbar discectomy. Clin Anat 2013; 26: 728-34.

14. Cramer GD, Skogsbergh DR, Bakkum BW, Winterstein JF, Yu S, Tuck NR Jr. Evaluation of transforaminal ligaments by magnetic resonance imaging. J Manipulative Physiol Ther 2002; 25: 199208.

15. Chun EH, Park HS. A Modified approach of percutaneous endoscopic lumbar discectomy (PELD) for far lateral disc herniation at L5-S1 with foot drop. Korean J Pain 2016; 29: 57-61. 